\title{
Annealing Cycle Dependence of Thermal Conductivity for Si-Ge-Au Thin Film Analyzed by Thermal Microscopy
}

\author{
Tsuyoshi Nishi, ${ }^{1 *}$ Tomohiro Mayama, ${ }^{1 * *}$ Hiromichi Ohta, ${ }^{1}$ and Yoichi Okamoto ${ }^{2}$ \\ ${ }^{1}$ Graduate School of Science and Engineering, Institute of Quantum Beam Science, \\ Ibaraki University, 4-12-1 Nakanarusawa, Hitachi, Ibaraki 316-8511, Japan \\ ${ }^{2}$ Department of Materials Science and Engineering, National Defense Academy, \\ 1-10-20 Hashirimizu, Yokosuka, Kanagawa 239-8686, Japan
}

(Received August 24, 2018; accepted November 9, 2018)

Keywords: thermal effusivity, $\mathrm{Si}-\mathrm{Ge}-\mathrm{Au}$, amorphous, thin film, thermal microscope, thermal conductivity

The thermal effusivity of a $\mathrm{Si}-\mathrm{Ge}-\mathrm{Au}$ thin film was measured using a thermal microscope. A thin-film sample with sixty-seven artificial intervals each comprising Si $(2.0 \mathrm{~nm}) / \mathrm{Au}$-doped Ge $(2.5 \mathrm{~nm})$ was prepared and annealed more than 20 times. The dependence of the thermal conductivity of the film on the number of annealing cycles was determined using the obtained experimental thermal effusivity data, bulk density, and specific heat capacity. The film thickness $(\sim 300 \mathrm{~nm})$ was greater than the thermal diffusion length of the samples. The thermal conductivity of the $\mathrm{Si}-\mathrm{Ge}-\mathrm{Au}$ thin film was satisfactory considering the number of annealing cycles. Thus, we elucidated the annealing effect of thermal conductivity for the $\mathrm{Si}-\mathrm{Ge}-\mathrm{Au}$ thin film.

\section{Introduction}

The heavy dependence of many countries on fossil fuels is a matter of serious public concern. One strategy to address this environmental crisis is to step up research on sustainable energy and to develop thermoelectric materials. The effectiveness of a material in thermoelectric applications is determined by the dimensionless figure of merit, ZT, which is expressed as ${ }^{(1)}$

$$
\mathrm{ZT}=S^{2} \sigma T / \lambda,
$$

where $S, \sigma, T$, and $\lambda$ are the Seebeck coefficient, electrical conductivity, absolute temperature, and thermal conductivity, respectively. A "good" thermoelectric material is defined as one with a large Seebeck coefficient, high electrical conductivity, and low thermal conductivity.

It is well known that good thermoelectric materials, which contain metal atoms and semimetal atoms, should possess the following structures: (1) a structure with a large number of atoms in the unit cell of crystals, (2) a superlattice structure, and (3) a structure containing large

\footnotetext{
*Corresponding author: e-mail: tsuyoshi.nishi.75@vc.ibaraki.ac.jp

** Corresponding author: e-mail: 18nm950g@vc.ibaraki.ac.jp

https://doi.org/10.18494/SAM.2019.2119
} 
atoms bonded loosely in the cage crystal structure of a large unit cell. Recently, Okamoto and others have suggested that the extremely high power of a $\mathrm{Si}-\mathrm{Ge}-\mathrm{Au}$ superlattice thin film is related to structural change and thermal conduction. ${ }^{(2-10)}$ On the other hand, we have measured the thermal effusivities of thin films by the thermoreflectance method using thermophysical microscopy (TM). ${ }^{(11)}$

With this background, the thermal effusivity of a $\mathrm{Si}-\mathrm{Ge}-\mathrm{Au}$ film was measured by TM. Moreover, the dependence of the thermal conductivity of the film on the number of annealing cycles was determined using the obtained experimental thermal effusivity data, bulk density, and specific heat capacity.

\section{Experimental Methods}

\subsection{Samples}

The detailed sample preparation procedure is described in Refs. 3-10. Thin films comprising Au-doped Ge layer and Si layer were prepared by an independent shutter operation with the simultaneous evaporation of these two components onto glass substrates in an ultrahigh-vacuum system. The base pressure of the stainless-steel chamber with a liquid $\mathrm{N}_{2}$ shroud was approximately $\sim 10^{-8} \mathrm{~Pa}$, but increased to up to $\sim 10^{-6} \mathrm{~Pa}$ during the operation of two electron beam guns. The evaporation rate and layer thickness were controlled by a computer with two sets of crystal oscillator thickness monitors. The deposition rate of both evaporation sources was maintained at approximately $0.05 \mathrm{~nm} / \mathrm{s}$. The crystal oscillator thickness monitors were calibrated using a surface morphology micrometer and X-ray low-angle diffraction system. The sample was prepared with sixty-seven artificial intervals each comprising $\mathrm{Si}$ (2.0 $\mathrm{nm}) / \mathrm{Au}$-doped $\mathrm{Ge}(2.5 \mathrm{~nm})$. One annealing cycle was performed at a heating rate of $10 \mathrm{~K} / \mathrm{min}$, after which the temperature was maintained at $673 \mathrm{~K}$ for $10 \mathrm{~min}$, cooled at $10 \mathrm{~K} / \mathrm{min}$, and then maintained at room temperature for $10 \mathrm{~min}$ in $\mathrm{N}_{2}$ gas flow at atmospheric pressure. The film thickness $(\sim 300 \mathrm{~nm})$ was greater than the thermal diffusion length of the samples. The structure of the $\mathrm{Si}-\mathrm{Ge}-\mathrm{Au}$ thin film is illustrated in Fig. 1.

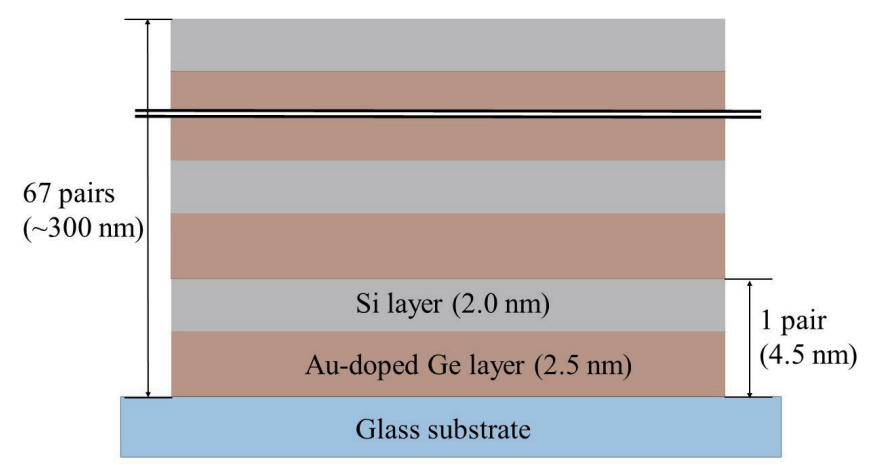

Fig. 1. (Color online) Schematic of structure of the $\mathrm{Si}-\mathrm{Ge}-\mathrm{Au}$ thin film. 


\subsection{Thermal effusivity measurements}

For the thermal effusivity measurements, it was necessary to sputter a Mo film, which was approximately $100 \mathrm{~nm}$ thick, onto the surface of the sample. Figure 2 shows the TM apparatus, which is described in detail in Ref. 12. Detailed information on typical TM and data processing for determining the thermal effusivity at one point of a sample is available in our previous publication; ${ }^{(13)}$ only a brief outline is provided here. The Mo thin film was first sputtered onto the sample to serve two functions: as a laser-beam refractor for temperature measurements and as a heat absorber. A small area of the sample surface was heated by an intensity-modulated laser beam, and the temperature response was monitored by another laser beam on the basis of thermoreflectance. The thermal effusivity was derived from the phase lag between the thermal wave and the thermoreflectance signal.

Each of the two laser beams used in the setup was coaxially aligned and focused on the sample surface. A compensating network based on a differential scheme was used to reduce the fluctuations caused by the instability of the probe laser. The diameters of the heating and detection areas were 23.4 and $7.2 \mu \mathrm{m}$, respectively. To justify the use of a one-dimensional heat-transfer model in the analysis of the measurement data, the area heated by the heating laser beam must be larger than the area used for the reflectivity measurements. The spatial resolution of the apparatus was determined from the sum of the diameter of the probing laser beam and the thermal diffusion length. In this study, the modulation angular frequency of the heating laser beam was $2 \mathrm{MHz}$. The sample surface could be viewed using a CCD camera built into the microscope's optical system. The heating laser beam with a sinusoidally modulated signal and angular frequency $\omega$ was incident on the sample surface. The temperature response of the

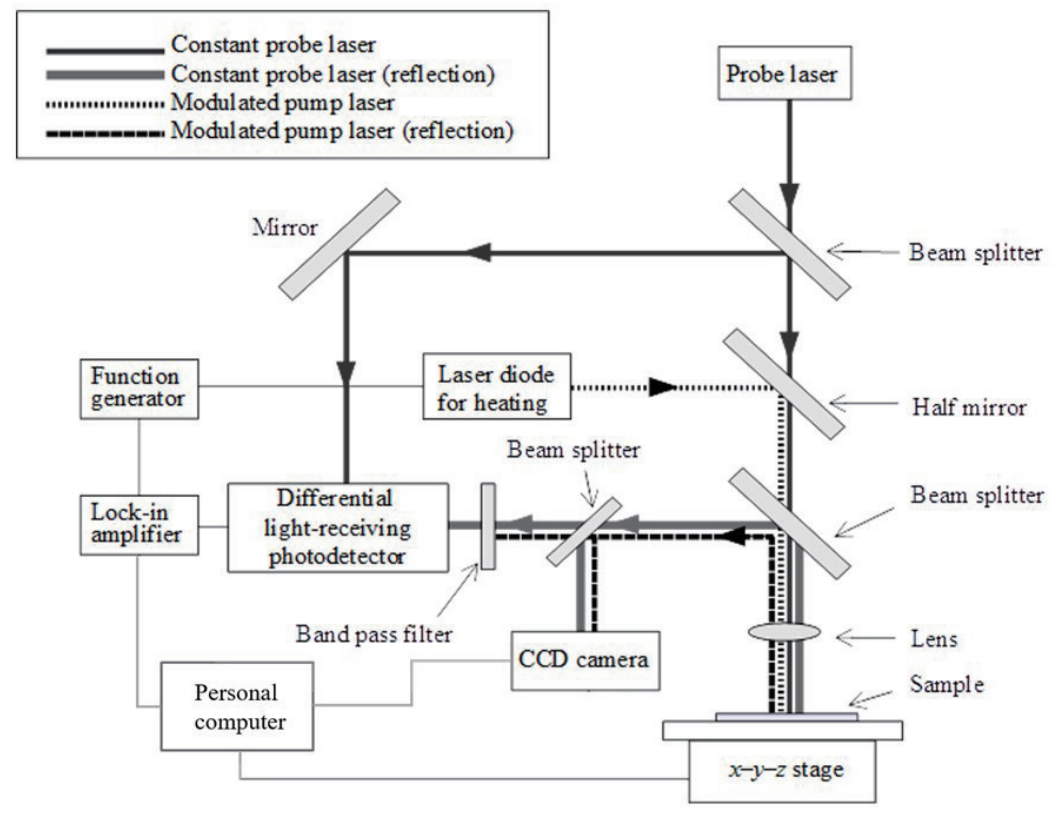

Fig. 2. Illustration of thermal microscope apparatus. 
sample surface to the heat input from the laser was measured by monitoring the variation in the intensity of the reflected beam of the second temperature-sensing laser that was operating at constant energy. The measurements were carried out at room temperature, and the temperature oscillated with a phase lag of $\theta$ relative to the phase of the heating laser. The thermal effusivity of the sample, $b_{s}$, was derived using Eqs. (2) to (4) below. The analytical formulation was based on the assumption that the contact thermal resistance of the coating/sample interface was negligible in a two-layered system and the temperature distribution in the sample reached a steady state. The analysis was based on one-dimensional heat flow in a semi-infinite layer:

$$
\theta=\frac{3}{4} \pi+\arctan \left(\frac{\cosh ^{2} \sqrt{\frac{\omega \tau_{f}}{2}}\left(\tanh \sqrt{\frac{\omega \tau_{f}}{2}}+\beta\right)\left(\tanh \sqrt{\frac{\omega \tau_{f}}{2}+\beta^{-1}}\right)}{\cos ^{2} \sqrt{\frac{\omega \tau_{f}}{2}}\left(\beta-\beta^{-1}\right) \tan \sqrt{\frac{\omega \tau_{f}}{2}}}\right),
$$

where

$$
\begin{aligned}
& \tau_{f}=d^{2} / \alpha_{f}, \\
& \beta=b_{s} / b_{f},
\end{aligned}
$$

and $\tau, \alpha$, and $d$ represent the characteristic time for heat diffusion, thermal diffusivity, and thickness of the Mo layer, respectively. The subscripts " $s$ " and " $f$ " are modifiers that indicate whether a variable represents a parameter of the sample or the Mo film, respectively. The value of $\theta$ in Eq. (2) was measured using a lock-in amplifier. The thermophysical properties of the Mo film and the values of $\alpha_{f}$ and $b_{f}$ were determined in separate experiments.

\subsection{Evaluation of thermal conductivity}

The thermal conductivity of the sample, $\lambda_{s}$, can be obtained from $b_{s}$ using Eq. (5) if the bulk density $\rho_{s}$ and the specific heat capacity $C_{p s}$ are known:

$$
\lambda_{s}=b_{s}^{2} / \rho_{s} C_{p s}
$$

The bulk density and specific heat capacity of the $\mathrm{Si}-\mathrm{Ge}-\mathrm{Au}$ thin-film sample are summarized in Table 1. The bulk density was evaluated on the basis of the additive law using

Table 1

Bulk density and specific heat capacity of the $\mathrm{Si}-\mathrm{Ge}-\mathrm{Au}$ thin-film sample.

\begin{tabular}{lcc}
\hline Sample & Bulk density $\left(\mathrm{kgm}^{-3}\right)$ & Specific heat capacity $\left(\mathrm{Jkg}^{-1} \mathrm{~K}^{-1}\right)$ \\
\hline $\mathrm{Si}-\mathrm{Ge}-\mathrm{Au}$ & 5767 & 300 \\
\hline
\end{tabular}




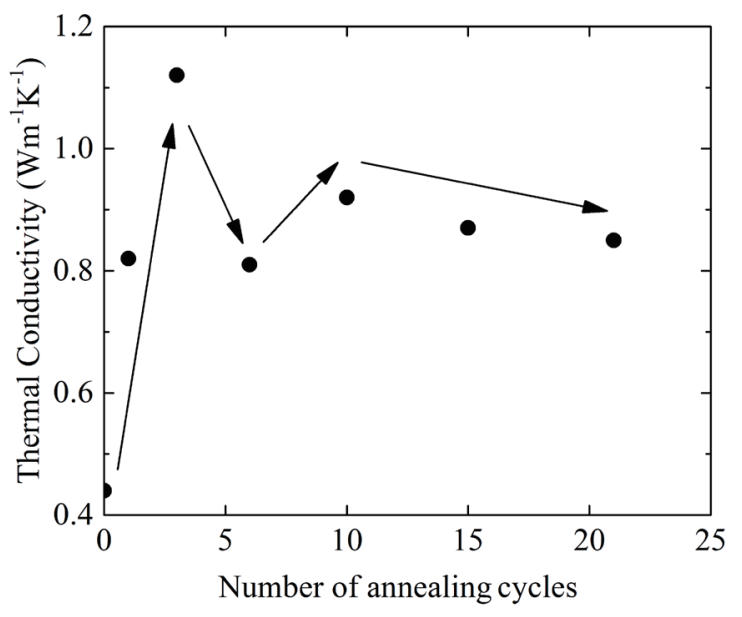

Fig. 3. Dependence of the thermal conductivity of $\mathrm{Si}-\mathrm{Ge}-\mathrm{Au}$ thin film on the number of annealing cycles.

the values for the solid state for $\mathrm{Si}, \mathrm{Ge}$, and $\mathrm{Au}$ in Ref. 14 at $298 \mathrm{~K}$. The specific heat capacity was evaluated on the basis of Kopp's law using values for the solid state for $\mathrm{Si}, \mathrm{Ge}$, and $\mathrm{Au}$ in Ref. 14 at 298 K. In this study, the mass fractions of the sample were 22, 70, and 8, respectively, for $\mathrm{Si}, \mathrm{Ge}$, and $\mathrm{Au}$.

\section{Results and Discussion}

Figure 3 shows the dependence of the thermal conductivity on the number of annealing cycles $(0-21)$ for the $\mathrm{Si}-\mathrm{Ge}-\mathrm{Au}$ thin film. For no annealing cycles, the thermal conductivity was relatively low, because the sample was in the form of an amorphous artificial superlattice thin film. For two annealing cycles, the thermal conductivity was relatively high, owing to the degradation of the superlattice. Following degradation, the superlattice became a simple amorphous thin film. The thermal conductivity decreased once for six annealing cycles, increased once for ten cycles, and slightly decreased for more than ten annealing cycles. This decrease in the thermal conductivity may be ascribed to the generation of a nanocrystalline nucleus, followed by a growth and increase in the number of nanocrystal grains. The thermal conductivity of the simple $\mathrm{Si}-\mathrm{Ge}-\mathrm{Au}$ amorphous thin film was approximately $1.1 \mathrm{Wm}^{-1} \mathrm{~K}^{-1}$, since the initial annealing led to the degradation of the artificial superlattice. Subsequently, the thermal conductivity decreased with increasing number of annealing cycles because of the phonon scattering effect due to the growth and increase in the number of nanocrystal grains. ${ }^{(2-10)}$

\section{Conclusions}

In this study, the dependence of thermal conductivity on the number of annealing cycles was determined for a $\mathrm{Si}-\mathrm{Ge}-\mathrm{Au}$ thin film. The following conclusions were drawn:

1. For no annealing cycles, the thermal conductivity was relatively low because the sample was an amorphous artificial superlattice thin film. 
2. The thermal conductivity slightly decreased with increasing number of annealing cycles for more than 10 cycles. This can be attributed to the nucleation and growth of nanocrystals.

\section{Acknowledgments}

We are grateful for the kind support of $\mathrm{H}$. Takiguchi, H. Miyazaki, and J. Morimoto from the National Defense Academy, and R. Okada and T. Nemoto from Ibaraki University. We thank T. Uno and H. Sato from PASONA Co., Ltd., for valuable discussions and comments.

\section{References}

1 D. M. Rowe: CRC Handbook of Thermoelectrics (CRC Press, New York, 1995).

2 Y. Okamoto, H. Uchino, T. Kawahara, and J. Morimoto: Jpn. J. Appl. Phys. 38 (1999) L945. https://doi. org/10.1143/JJAP.38.L945

3 H. Uchino, Y. Okamoto, T. Kawahara, and J. Morimoto: Jpn. J. Appl. Phys. 39 (2000) 1675. https://doi. org/10.1143/JJAP.39.1675

4 S. M. Lee, Y. Okamoto, T. Kawahara, and J. Morimoto: Proc. 20th Int. Conf. Thermoelectrics (2001) 348.

5 K. Fukushima and N. Kondo: Jpn. J. Appl. Phys. 40 (2001) 3226. https://doi.org/10.1143/JJAP.40.3226

6 Y. Okamoto, H. Fujita, T. Kawahara, S. M. Lee, and J. Morimoto: Proc. 19th Int. Conf. Thermoelectrics (2000) 435.

7 T. Kawahara, H. Fujita, S. M. Lee, Y. Okamoto, and J. Morimoto: Proc. 19th Int. Conf. Thermoelectrics (2000) 458.

8 K. Fukui, M. Nakamori, Y. Okamoto, and J. Morimoto: Proc. 23rd Int. Conf. Thermoelectrics (2005) 172.

9 Y. Okamoto, A. Miyata, Y. Sato, H. Takiguchi, T. Kawahara, and J. Morimoto: Jpn. J. Appl. Phys. 42 (2003) 3048. https://doi.org/10.1143/JJAP.42.3048

10 A. Miyata, Y. Okamoto, H. Takiguchi, T. Kawahara, and J. Morimoto: Trans. Mater. Res. Soc. Jpn. 29 (2004) 2801. https://doi.org/10.1143/JJAP.46.4622

11 T. Nishi, S. Yamamoto, M. Okawa, K. Hatori, T. Ikeda, and H. Ohta: Thermochim. Acta 659 (2018) 39. https:// doi.org/10.1016/j.tca.2017.11.004

12 H. Ohta, K. Hatori, G. Matsui, T. Yagi, S. Miyake, T. Okamura, R. Endoh, R. Okada, K. Morishita, S. Yokoyama, K. Taguchi, and H. Kato: Meas. Sci. Technol. 27 (2016) 115002. https://doi.org/10.1088/0957-0233/27/11/115002

13 K. Hatori, T. Baba, N. Taketoshi, and H. Ohta: Rev. Sci. Inst. 76 (2005) 114901. https://doi. org/10.1063/1.2130333

14 Shinpen Netubussei Handbook, ed. Japan Society of Calorimetry and Thermal Analysis (Yokendo, Tokyo, 2008) p. 20 (in Japanese).

\section{About the Authors}

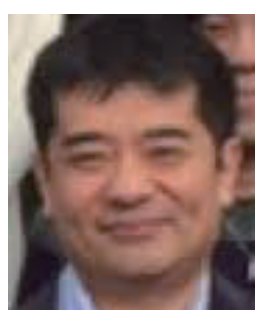

Tsuyoshi Nishi received his B.S. degree from Tokyo University of Science, Japan, in 1999 and his M.S. and Ph.D. degrees from Tohoku University, Japan, in 2001 and 2004, respectively. From 2004 to 2015, he was a research scientist at the Japan Atomic Energy Agency (JAEA), Japan. Since 2015, he has been an associate professor at Ibaraki University. His research interests are on thermophysical properties, structural analysis, noncrystal materials, and nuclear materials. (tsuyoshi.nishi.75@vc.ibaraki.ac.jp) 


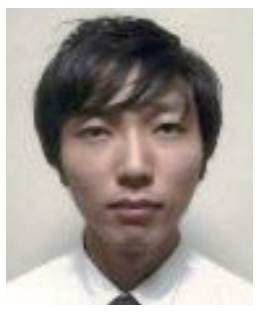

Tomohito Mayama received his B.S. degree from Ibaraki University, Japan, in 2018. Since 2018, he has been an M.S. student at Ibaraki University. His research interests include the laser flash method, thermal microscopy, plastic materials, and thermoelectric materials.(18nm950g@vc.ibaraki.ac.jp)

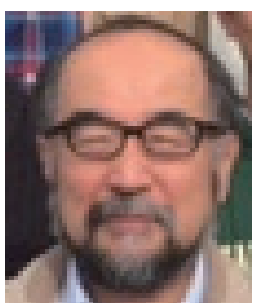

Hiromichi Ohta received his B.S., M.S., and Ph.D. degrees from Tohoku University, Japan, in 1978, 1980, and 1984, respectively. From 1984 to 2008, he was a research associate and an associate professor at Ibaraki University, Japan. Since 2008, he has been a professor at Ibaraki University. His research interests include thermophysical properties, computational material science, composite materials, and silicate melts.

(hiromichi.ohta.ys@vc.ibaraki.ac.jp)

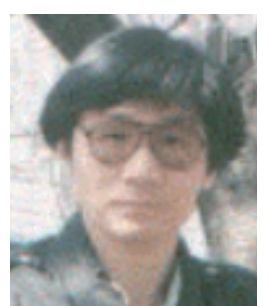

Yoichi Okamoto received his B.S., M.S., and Ph.D. degrees from the University of Tsukuba, Japan, in 1981, 1983, and 1990, respectively. From 1986 to 1997, he was a research associate and an assistant professor at the National Defense Academy (NDA) of Japan, and since 1997, has been an associate professor there. His research interests are on thermoelectric materials, thermal measurement, and superlattice thin films.

(rshow@nda.ac.jp) 